\title{
Arc length coding by interference of theta frequency oscillations may underlie context-dependent hippocampal unit data and episodic memory function
}

\author{
Michael E. Hasselmo ${ }^{1}$ \\ Center for Memory and Brain, Department of Psychology and Program in Neuroscience, Boston University, \\ Boston, Massachusetts 02215, USA
}

\begin{abstract}
Many memory models focus on encoding of sequences by excitatory recurrent synapses in region CA3 of the hippocampus. However, data and modeling suggest an alternate mechanism for encoding of sequences in which interference between theta frequency oscillations encodes the position within a sequence based on spatial arc length or time. Arc length can be coded by an oscillatory interference model that accounts for many features of the context-dependent firing properties of hippocampal neurons observed during performance of spatial memory tasks. In continuous spatial alternation, many neurons fire selectively depending on the direction of prior or future response (left or right). In contrast, in delayed non-match to position, most neurons fire selectively for task phase (sample vs. choice), with less selectivity for left versus right. These seemingly disparate results are effectively simulated by the same model, based on mechanisms similar to a model of grid cell firing in entorhinal cortex. The model also simulates forward shifting of firing over trials. Adding effects of persistent firing with reset at reward locations addresses changes in context-dependent firing with different task designs. Arc length coding could contribute to episodic encoding of trajectories as sequences of states and actions.
\end{abstract}

Many models of the hippocampus focus on strengthening of synaptic connections between neurons to encode associations between sequential patterns of neural activity representing items or locations (McNaughton and Morris 1987; Treves and Rolls 1994; Jensen and Lisman 1996a; Levy 1996; Tsodyks et al. 1996; Wallenstein and Hasselmo 1997; Hasselmo 2005b; Hasselmo and Eichenbaum 2005; Howard et al. 2005). For example, in one previous model, synaptic retrieval of sequences was used to model context-dependent firing properties in a continuous spatial alternation task (Hasselmo and Eichenbaum 2005) in which neurons fire selectively dependent on prior or future direction of response, for example, firing on the stem of a T-maze after a right but not a left turn trial (Wood et al. 2000). However, recent experiments on the firing properties of hippocampal neurons have produced data with features not explained by models using synaptic modification to store sequential associations. In particular, neurons firing in a continuous spatial alternation task show a progressive forward shift of firing location toward reward location (Lee et al. 2006), whereas models of synaptic modification primarily predict a backward shift of firing location (Blum and Abbott 1996; Mehta et al. 2002). In addition, neural firing in continuous spatial alternation differs from data in a delayed nonmatch to position (DNMP) task that shows contextual firing specific to task phase (sample vs. choice), which appears to depend less on the left or right direction of the response (Griffin et al. 2007).

As shown here, these different patterns of neural activity in continuous spatial alternation and DNMP are effectively modeled using a new theoretical framework. Recent experimental data and computational modeling (Burgess et al. 2007; Giocomo et al. 2007) suggest an alternate mechanism that may contribute

\footnotetext{
'Corresponding author.
}

E-mail hasselmo@bu.edu; fax (617) 358-3296.

Article is online at http://www.learnmem.org/cgi/doi/10.1101/lm.686607. to encoding the sequential order of information in space and time. This mechanism uses interference between theta frequency oscillations, which could include both network theta oscillations (Buzsaki et al. 1983; Stewart and Fox 1990; Brankack et al. 1993; Vertes and Kocsis 1997; Lengyel et al. 2003; Hasselmo 2005b; O'Keefe and Burgess 2005) and intrinsic subthreshold membrane potential oscillations shown in single neurons of entorhinal cortex (Alonso and Llinas 1989; Alonso and Klink 1993; White et al. 1995; Dickson et al. 2000; Lengyel et al. 2003; Fransén et al. 2004; Giocomo et al. 2007). Recent data show that intrinsic membrane potential oscillations differ in frequency for stellate cells at different positions along the dorsal-to-ventral axis of the medial entorhinal cortex (Giocomo et al. 2007; Hasselmo et al. 2007). These frequency differences support a model (Burgess et al. 2005, 2007), further analyzed in Giocomo et al. (2007) and Hasselmo et al. (2007), accounting for differences in the spatial periodicity of grid cell firing responses at different positions along the dorsalto-ventral axis of the entorhinal cortex (Hafting et al. 2005; Sargolini et al. 2006). Further intracellular recording data show that neurons in layer III of the entorhinal cortex have persistent firing activity with frequencies that differ between individual neurons, but are consistent within a neuron and fall within the theta frequency range (Tahvildari et al. 2007). The model also makes use of data showing changes in theta frequency and neural firing rate associated with running speed (Taube et al. 1990; Sharp 1996; O'Keefe et al. 1998; Maurer et al. 2005; Sharp and TurnerWilliams 2005; Sharp et al. 2006; Geisler et al. 2007).

The model presented here shows how interference between theta rhythm periodicity of afferent input from entorhinal neurons and theta rhythm periodicity of hippocampal circuits can effectively replicate the neural firing observed experimentally in spatial alternation and DNMP tasks, using the same parameters in simulations of both behavioral tasks. This provides further support for the oscillatory interference mechanism already 
shown to model grid cell firing properties in entorhinal cortex (Burgess et al. 2005, 2007; Giocomo et al. 2007; Hasselmo et al. 2007) and theta phase precession in hippocampal neurons (O'Keefe and Recce 1993; Bose et al. 2000; Bose and Recce 2001; Lengyel et al. 2003; Burgess et al. 2005, 2007; O'Keefe and Burgess 2005). This model differs in structure from models of theta phase precession based on synaptic retrieval (Jensen and Lisman 1996a; Tsodyks et al. 1996; Wallenstein and Hasselmo 1997; Hasselmo and Eichenbaum 2005), but is not incompatible with those models.

The model presented here effectively simulates (1) the context-sensitivity of neuronal firing during spatial alternation (Wood et al. 2000); (2) the forward shift of firing activity toward goal locations (Lee et al. 2006); (3) the task phase selectivity of firing in a DNMP task (Griffin et al. 2007); and (4) differences in firing in spatial alternation with different reward structures (Bower et al. 2005). In addition, the basic model is incorporated in a preliminary model that encodes trajectories by modification of connections between context-dependent place cells and neurons showing modulation by speed and head direction.

\section{Results}

In the model presented here, interference between theta frequency oscillations can account for the spiking behavior of hippocampal neurons in behavioral tasks in the T-maze including continuous spatial alternation and delayed non-match to position. The correct performance in these behavioral tasks is shown in Figure 1. In continuous spatial alternation (Fig. 1A), consider a rat starting at the left reward location. The rat runs down along the left return arm, runs up the central stem of the T-maze, and into the right reward arm (trajectory shown in red). After getting the reward, the rat runs back down on the right return arm, runs up the central stem, and into the left reward arm to receive the reward (trajectory shown in blue).
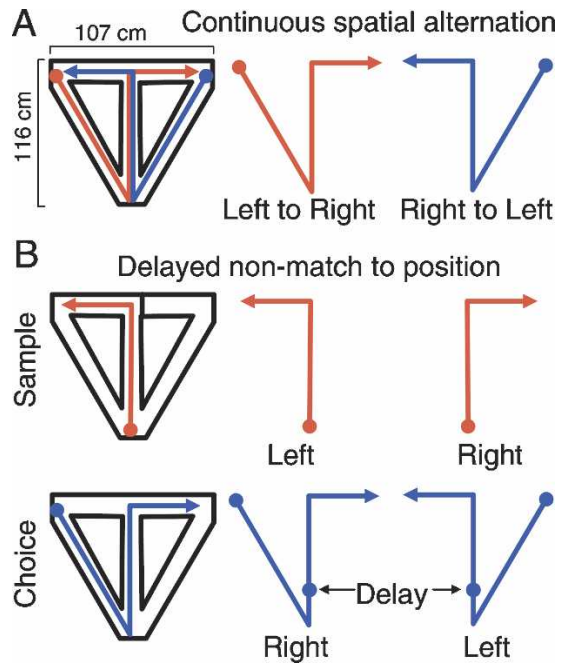

Figure 1. ( $A$ ) Correct behavior in continuous spatial alternation. The rat runs up the stem into one reward arm (e.g., the left) and receives a food reward. The red trajectory shows how the rat must run back on the left return arm and up the stem into the right arm for a reward; the blue trajectory shows how it must then run back on the right return arm and up the stem into the left arm for a reward. The rat must alternate between these two trajectories. (B) Correct behavior in DNMP. Red trajectories show that on sample trials, the rat runs up the stem and is only allowed into one reward arm (either left or right). Blue trajectories show that on a subsequent choice trial, the rat runs back on the return arm, waits at the delay position (circle) until the block is removed, and then runs up the stem into the opposite arm.
In delayed non-match to position (Fig. 1B), behavior is split into sample and choice phases. On each sample phase, one reward arm is blocked. The rat runs up the central arm (trajectory in red) and into the unblocked reward arm (left or right). On the following choice phase (shown in blue), a block is placed on the stem. The rat runs along the return arm to wait at the base of the stem during the delay, then, when the block is removed, it runs into the reward arm opposite to the sample arm. For example, a rat might perform the following sequence: left sample, right choice, right sample, left choice.

\section{Oscillatory interference computes arc length}

The simulation of neural activity in these tasks results in the neural computation of arc length-that is, the length of an irregular trajectory through the environment. As described in Materials and Methods, modulation of the frequency of oscillations by running speed in the simulations causes oscillatory interference with a magnitude that depends on the arc length of the trajectory run by the simulated rat. This oscillatory interference is proposed to occur between speed-modulated oscillations in entorhinal cortex and oscillations paced at a different frequency by septal input to the hippocampal subregions. Because the frequency of entorhinal oscillations is determined by running speed on each segment of the trajectory, the phase of oscillatory interference is proportional to the sum of all the previous individual segments of movement along that trajectory. This approximates the arc length of the trajectory at the resolution determined by the sampling rate of position (every $20 \mathrm{msec}$ in these simulations). Therefore, for a given set of neurons, the phase of oscillatory interference will depend on the arc length of the trajectory traveled by the simulated rat since the start of oscillations in that set of neurons. Note that arc length differs from the computation of two-dimensional distance within the task. Arc length is the length of a particular irregular path between two points that includes all turns and deviations, rather than the length of a straight line (Euclidian distance) between those two points. Mechanisms for causing cell firing dependent on arc length in the simulations are described further in Materials and Methods. The use of activity dependent on running speed is supported by data showing effects of running speed (translational motion) on neuronal firing in postsubiculum (Taube et al. 1990; Sharp 1996), deep layers of entorhinal cortex (Sargolini et al. 2006), axons in the hippocampus (O'Keefe et al. 1998), and in subcortical structures including the medial mammillary nucleus and habenula (Sharp and Turner-Williams 2005; Sharp et al. 2006).

\section{Continuous spatial alternation}

As shown in Figure 2A, neural activity in continuous spatial alternation shows strong specificity for left-to-right (L to R) versus right-to-left ( $\mathrm{R}$ to $\mathrm{L}$ ) trial type. Initial studies in spatial alternation tasks show many neurons with strong differential firing (Frank et al. 2000; Wood et al. 2000). These cells are sometimes referred to as "splitter" cells, as they split their response differentially between different trial types. In a recent study (Lee et al. 2006), out of 78 neurons that fired in spatial locations in the stem of the task, 53 neurons showed significant selectivity for one trial type versus the other (left vs. right). Figure $2 \mathrm{~A}$ shows examples of two of these neurons (Cell r3-d4-tt6-cl1 and Cell r2-d1-tt2-cl4 from Fig. 1 in Lee et al. 2006). The firing rate of the neuron in the stem is shown next to each trace.

The simulation presented here effectively replicates the selectivity of firing in the continuous spatial alternation task, using oscillatory interference with phase dependent on the arc length of the rat's trajectory. The position of firing in the task depends on the phase of entorhinal neuronal firing relative to hippocam- 


\section{Experimental data}

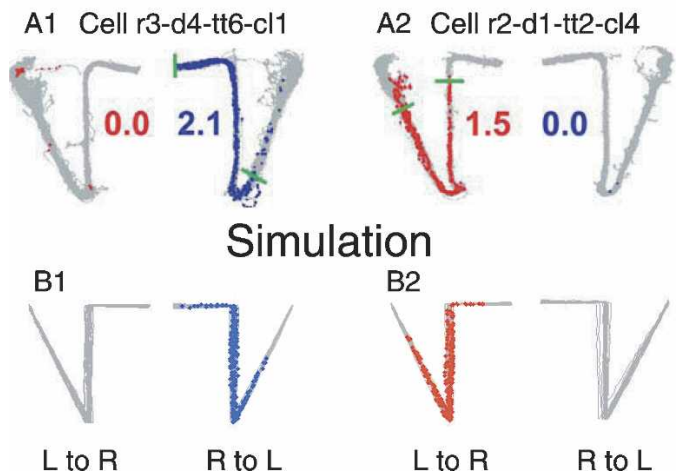

Figure 2. (A) Activity of splitter cells recorded during continuous spatial alternation. The data are reprinted with permission from Elsevier $(2) 2006$, Lee et al. (2006). (A1) Cell r3-d4-tt6-cl1 fires very little when the rat runs from the left return arm through the stem to the right reward arm ( $L$ to $\mathrm{R}$ ), but fires extensively (blue dots) when the rat runs from the right return arm through the stem to the left reward arm ( $R$ to $L)$. Numbers indicate the firing rate in the stem during the two trial types. (A2) Cell r2-d1-tt2-cl4 fires extensively (red dots) when the rat runs from the left return arm to the right reward arm, but fires very little when the rat runs from the right return arm to the left reward arm. (B) Simulations show the same pattern of selective firing found in the experimental data. (B1) The simulation shows firing on $\mathrm{R}$ to $\mathrm{L}$ trials (blue dots) using entorhinal input with the phase at $64.3^{\circ}$. (B2) The simulation shows selective firing on $L$ to $\mathrm{R}$ trials (red dots), using the same parameters with the starting phase $\varphi$ of entorhinal input shifted by $180^{\circ}$ (phase $244.3^{\circ}$ ).

pal theta rhythm. In Figure 2B1, the simulated rat starts out at the center of the stem in a right-to-left trial type with the simulated cell receiving entorhinal input at a specific phase $(1.122$ radians, $64.3^{\circ}$ ). The firing of spikes by the simulated cell is shown with blue dots indicating the location of the virtual rat at the time of each spike. The spiking activity consistently occurs in the stem on right-to-left trials (Cell r3-d4-tt6-cl1 simulation), and spiking does not occur on left-to-right trials.

In contrast, Figure $2 \mathrm{~B} 2$ shows a different simulated cell with a phase shift that differs from the simulation on the left by $180^{\circ}(\pi$ radians), for a phase of $244.3^{\circ}$. Note that both simulations used the same starting location of the virtual rat. This separate simulated cell shows firing (red dots) that consistently occurs when the virtual rat is on left-to-right trials, therefore more strongly resembling the data on firing specificity for Cell r2-d1-tt2-cl4. Thus, the simulation effectively replicates the trial-type selectivity in continuous spatial alternation. The position of the firing field in the task depends on the starting phase of entorhinal input relative to hippocampal theta rhythm oscillations.

The simulation of splitter cell firing used entorhinal input with the same baseline frequency as hippocampal theta rhythm $(6 \mathrm{~Hz})$. The interference pattern is influenced by the arc length of the rat's trajectory in the task rather than time because entorhinal activity is modulated by speed of movement of the simulated rat, which varies around the same average speed as the real rat (26 $\mathrm{cm} / \mathrm{sec}$ ). Thus, this simulation suggests that the response pattern seen in experimental data could indicate an arc length cell. See further discussion in the Materials and Methods section.

\section{Forward shifting toward goal location}

As shown in Figure 3, the model also accounts for an additional property of experimental data on neural firing during performance of continuous spatial alternation. The distribution of firing in the examples shown in Figure $2 \mathrm{~A}$ shows relatively large firing fields. The large size of the firing fields is partly due to a progressive forward shift of firing location in the task toward the reward location over trials (Lee et al. 2006). The top of Figure 3 shows an example of a single neuron (r2-d4-tt6-cl1 from Fig. 2 in Lee et al. 2006) that shows a strong progressive forward shift over 40 trials of continuous spatial alternation. Firing starts in the right return arm on early trials, shifts forward to positions on the stem around trial 25, and reaches the start of the left reward arm by trial 40 . This neuron shows an average shift of $\sim 4 \mathrm{~cm}$ per trial. Across the full population of neurons with firing fields in the stem, there is a robust and consistent forward shift of $\sim 1 \mathrm{~cm} /$ trial (Lee et al. 2006).

The simulation can account for the forward shifting of neural activity over trials, as shown in the bottom of Figure 3. The same parameters are used for the simulation in Figure 3 as for Figure 2B1, but the plotting of spikes is separate for different sets of trials in Figure 3. As can be seen here, the firing field of the simulated cell starts in the right return arm (owing to the starting phase of $64.3^{\circ}$ ). Over trials, the firing of spikes relative to the location of the simulated rat gradually shifts forward into the stem and eventually reaches the start of the left reward arm. This forward shift occurs because of a small difference between the arc length of one cycle of the task and the arc length between firing locations caused by interference between oscillations. In a sense, this resembles a larger-scale precession of neural firing relative to task phase (as shown in Fig. 5, below).

\section{Delayed non-match to position (DNMP)}

The same simulation can effectively replicate what initially appeared to be a very different firing pattern in DNMP. In the experiment testing firing activity in the DNMP task, we initially expected to see firing similar to the continuous spatial alternation task, with selectivity for left-versus-right trials in either the sample or the choice phase or possibly both (Hasselmo 2005b; Hasselmo and Eichenbaum 2005). As shown in Figure 4A, the pattern of activity in many neurons in the DNMP task did not seem to correspond to the pattern seen in the continuous spatial alternation task (Griffin et al. 2007). Instead, the majority of cells showed selective responding based on the task phase (sample vs. choice), with less dependence on whether the task required left or right responses. The example cell shown in Figure $4 \mathrm{~A}$ is sample-selective. It generates spikes (red dots) at locations throughout the sample phase of the task regardless of turn direction (left sample, right sample). In contrast, during the choice

\section{A. Experimental Data (Cell r2-d4-tt6-cl1)}

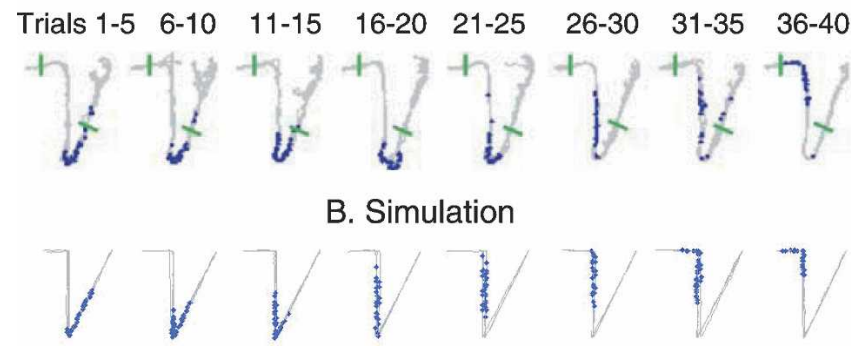

Figure 3. (A) Experimental data show forward shifting of the firing location of a neuron (r2-d4-tt6-cl1) during continuous spatial alternation. The data are reprinted with permission from Elsevier $\odot$ 2006, Lee et al. (2006). The cell fires selectively when the rat runs from the right return arm through the stem to the left reward arm ( $R$ to $L$ ). Over trials, the location of firing gradually shifts toward the reward location. In early trials $(1-5)$, firing occurs in the right return arm. By late trials (35-40), firing is at the start of the left reward arm. (B) Simulation of forward shifting during continuous spatial alternation. Firing of the cell from Figure $2 B 1$ is split into individual sets of five trials to show the forward shift that occurs over trials, due to the difference in spatial period of the oscillatory interference versus the arc length of the task. 


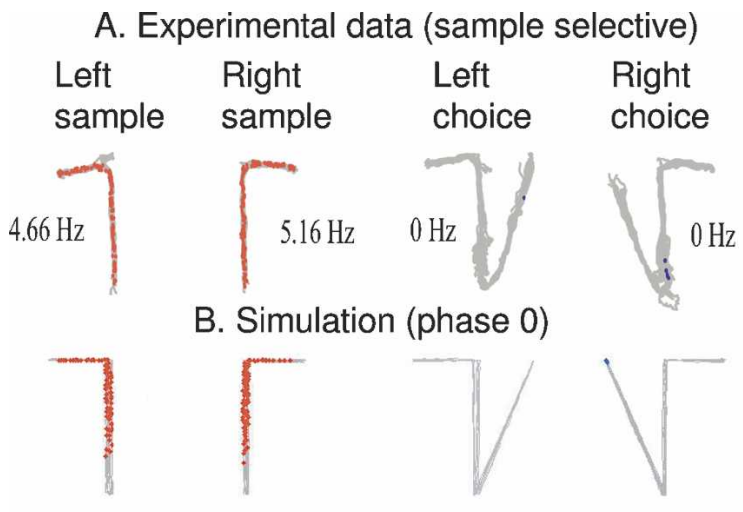

C. Experimental data (choice selective)

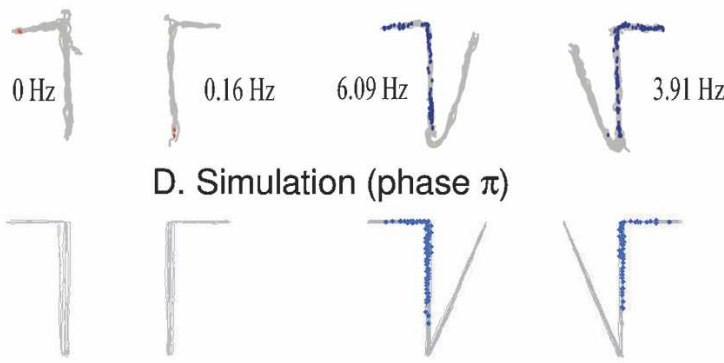

Figure 4. (A) Experimental data showing the task phase selectivity during DNMP. The cell shows firing (red dots) at locations throughout the sample phase of the task (left sample, right sample). During the choice phase, no firing is observed (left choice, right choice). The data are reprinted with permission from The Society for Neuroscience $\odot$ 2007, Griffin et al. (2007). (B) Simulation effectively shows the same task phase selectivity as the experimental data. With the phase of entorhinal input set at phase zero, the simulated neuron fires on both left and right sample trials (red dots), but does not fire during the choice phase. (C) Data from a different cell showing selective firing on choice phases (blue dots) but not on sample phase trials (Griffin et al. 2007). (D) In simulations, setting the phase of entorhinal input at $180^{\circ}$ (phase $\pi$ ) results in selective firing on both left and right choice trials (blue dots), but no firing during the sample phase.

phase, no firing is observed (left choice, right choice). Figure 4C shows an example of a choice-selective cell. This cell generates spikes at locations throughout the choice phase, regardless of it being a left or right choice trial (left choice, right choice), but does not respond at all during sample trials.

Our previous model predicted splitter cells because of retrieval of activity representing left or right responses on the sample trial (Hasselmo 2005b; Hasselmo and Eichenbaum 2005). In contrast, the model based on oscillatory interference can account for the firing in DNMP with the same parameters used for continuous spatial alternation. All that was required was programming the virtual rat to move in a pattern corresponding to the DNMP task. Then different patterns of firing in sample versus choice depended only on the starting phase of the oscillation. As shown in Figure 4B, when the phase of oscillation is set at zero in the model, the simulated spiking activity (red dots) occurs selectively in the sample phase of the task (left sample, right sample) and does not occur during the choice phase. In contrast, as shown in Figure 4D, when the oscillation is started at a phase that differs by $\pi$ radians $\left(180^{\circ}\right)$, the simulated spiking activity (blue dots) occurs selectively in the choice phase of the task (left choice, right choice) and does not occur in the sample phase. The forward shifting that appears during continuous alternation (Fig. 3) also appears to a smaller degree in the DNMP data (Griffin et al. 2007), and appears in the model of these data. (Plotting across multiple trials obscures this effect.)

As shown in Figure 5, the simulations use the same mechanism to replicate the trial-specific firing that occurs during continuous alternation and the task-phase-specific firing in data from DNMP. Interference at regular intervals of arc length could cause selective firing for one direction of response (e.g., left turn trials) in continuous alternation. The same periodicity could cause selective firing for only one task phase (e.g., the choice phase) in the DNMP task. Thus, this new model provides a simple framework that effectively accounts for both sets of data with the same model in Figures 2-4.

\section{Reward/stopping locations influence context-dependent firing}

A further modification of the model can account for additional features of the data. A recent study demonstrated that contextdependent firing of neurons may depend on the location of the reward (Bower et al. 2005). In that study, selective contextdependent firing was shown for overlapping segments of trajectories with different prior trajectories. However, the selective firing did not occur if the rat stopped for the reward at the start of the overlapping segment. This suggests that stopping and/or receiving the reward at the start of the overlapping segment somehow prevented firing dependent on the preceding segment of a trajectory. This could be due to theta rhythm oscillations being shut down when the rat stops at a reward location. The theta rhythm shows reductions at reward locations and decreases even as the rat is approaching a reward location (Wyble et al. 2004). The temporary decrease in theta rhythm could result in periodicity being dependent on the latest reward location. Note that stopping per se does not have to reset the theta rhythm, as stopping at non-reward locations causes less suppression of theta rhythm (Wyble et al. 2004).

The modified model involved changes in the pattern of activity at each reward location. When the virtual rat reached a reward location, the previously active entorhinal neurons would stop firing, and this would terminate oscillations. Then, when the virtual rat left the reward location, a different population of neurons selective to that location would start firing persistently and drive oscillatory interference with a phase dependent on arc length from the specific reward location. The use of persistent

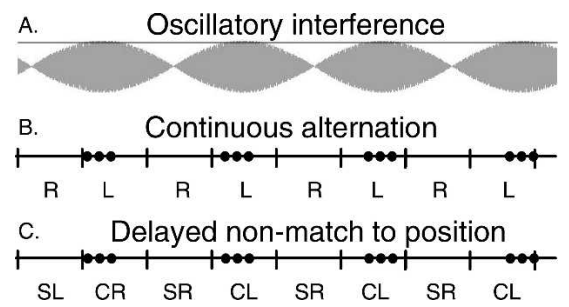

Figure 5. Periodicity of oscillatory interference can simulate neuronal firing in the two tasks. (A) Sum of hippocampal and entorhinal oscillations with different frequencies. Interference causes changes in amplitude that cross the firing threshold (line) at regular spatial intervals. (B) In continuous alternation, the position of the rat along the one-dimensional track shows spatial periodicity between left trials (L) and right trials (R). Speedmodulated oscillatory interference results in spatially periodic firing (black dots) that only falls in one trial type (L). The slightly longer arc length relative to spatial periodicity of the task results in gradual forward shifting of firing in the task. (C) Spatial periodicity in DNMP. The position of the rat along a one-dimensional plot of movement shows periodicity between sample and choice trials, varying between sample left (SL) and sample right (SR), and choice left (CL) and choice right (CR) trials. The same periodicity that causes selective $L$ versus $R$ trial response in alternation causes selective task phase response in DNMP (here firing occurs in choice trials). 
A. Reset at left reward

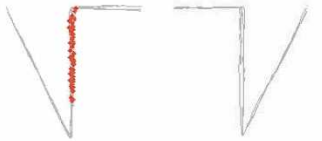

B. Reset at base of stem

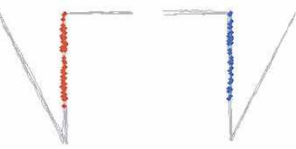

$\mathrm{L}$ to $\mathrm{R} \quad \mathrm{R}$ to $\mathrm{L}$

Figure 6. Modification of model by including reset of persistent spiking in different populations of entorhinal neurons. $(A)$ When persistent spiking is reset to activate a location-specific population at the reward location in the left arm (left reward), the firing shows selectivity for left-toright versus right-to-left trials. (B) When persistent spiking is reset to activate a location-specific population at a reward location at the base of the stem, firing occurs for both left and right trials, consistent with data showing lack of trial selectivity after reward at the base of the stem.

activity is supported by neurophysiological data showing persistent firing at theta frequencies for many minutes due to afterdepolarization currents in neurons of entorhinal cortex layer II and layer III (Klink and Alonso 1997a,b; Fransén et al. 2002; Tahvildari et al. 2007).

As shown in Figure 6, the modified model effectively accounts for the effects of stopping at reward locations. If the virtual rat stops at the base of the stem (Fig. 6B), then all neurons show equivalent firing in the stem (non-splitters). If the virtual rat stops at the end of a reward arm (Fig. 6A), but not at the base of the stem, then different entorhinal neurons are activated for the different stopping locations (splitters). This results in some hippocampal neurons showing selective firing in the stem for $\mathrm{L}$ to $\mathrm{R}$ trials. Separate hippocampal neurons receiving input from a different set of entorhinal neurons show selective firing in the stem for R to L trials. This is consistent with the data on effects of reward at the start of the overlapping segment (Bower et al. 2005).

\section{Model of episodic memory for trajectories}

These simulations support the idea that context-dependent firing of hippocampal neurons in tasks performed on a linear track could result from interference between oscillations. When interference has a spatial periodicity that matches the full arc length of the task (Fig. 5), this appears as context-dependent firing. These properties differ from place cells and grid cells that fire relatively consistently each time a rat is in a specific spatial location. The firing of context-dependent neurons appears to depend on arc length of the task trajectory rather than twodimensional position. This raises the question of the functional role of such periodicity.

These context-dependent responses could play a role in the episodic encoding and retrieval of trajectories through the environment, specifically enhancing function by moving beyond purely spatial representations. This section first describes a potential mechanism using grid cells, place cells, and head direction cells for episodic encoding and retrieval of trajectories. Subsequently, this section describes how context-dependent cells (that appear to compute arc length rather than place) could enhance episodic encoding and retrieval of trajectories.

As summarized in Figure 7A, grid cells, place cells, and head direction cells could interact for encoding and retrieval of trajectories. This involves three stages of functional projections: (1) from head direction cells to grid cells; (2) from grid cells to place cells; and (3) from place cells to head direction cells. See Materials and Methods for model equations. During encoding, head direction cell activity driven by actual movement updates the grid cell firing pattern, which updates the place cell activity. Episodic encoding of the trajectory then results from modification of synapses connecting place cells to active head direction cells. During retrieval, these modified synapses allow each place cell representation to activate an associated pattern of head direction cell activity, allowing each state (location) to retrieve the associated action that retrieves a subsequent state.

As shown in Figure 7D, the above mechanism works effectively for retrieving the initial nonoverlapping segment of a trajectory. The trajectory during encoding is shown in light gray, with arrows indicating the direction of movement. This is the trajectory of a real rat in experimental data from Hafting and Fyhn in the Moser lab (Hafting et al. 2005). During retrieval, the starting location (Start) activates head direction cells that update the grid cell representation to activate a new set of place cells a short distance along the retrieved trajectory. This new set of place

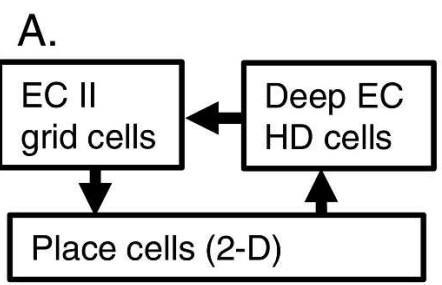

B.

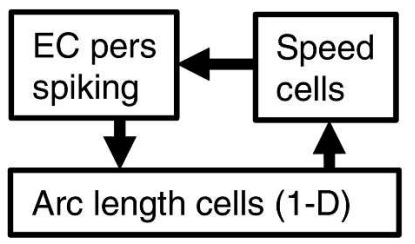

C.

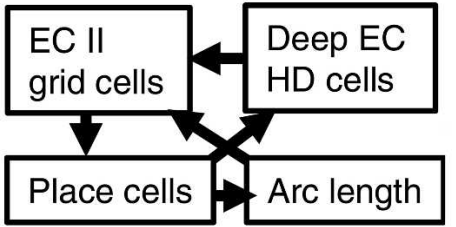

D. Place-HD

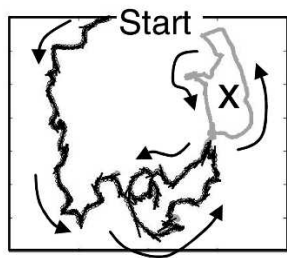

E. Arc length

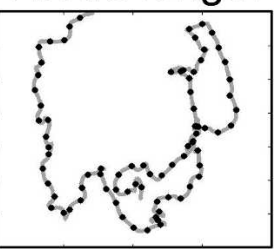

F. Combined

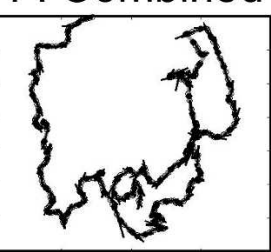

Figure 7. (A) Circuit for trajectory encoding. Head direction cells drive grid cells in entorhinal cortex layer II (EC II), which drive hippocampal place cells. During encoding, synapses are strengthened between active place cells (state) and speed-modulated head direction cells (action). During retrieval, place cells activate head direction cells coding the velocity from that location, which then drive grid cell phase changes to activate place cells coding the next location. (B) Arc length circuit. Movement speed cells alter the frequency of persistent spiking entorhinal neurons (EC pers spike). Oscillatory interference drives neurons responding on the basis of arc length along the trajectory. Arc length cells activate associated speed cells. (C) Combination of the grid-place-HD circuit and the pers-arc-speed circuit. $(D)$ The trajectory during encoding is plotted in gray. Arrows show the direction of movement during encoding and of activity spread during retrieval. Place cells (open circles) activated sequentially during retrieval drive head direction activity (line segments) that updates grid cell phase to retrieve the next place cell. Retrieval accurately covers the trajectory on the left, but the loop labeled with an X is not retrieved because overlap causes retrieval to skip to a later segment. $(E)$ Circuit 7B activates arc length cells (black circles) at regular intervals along the encoded trajectory (gray line) regardless of overlap. ( $F$ ) With the combined circuit, arc length cells reset the grid cell phase correctly after the overlapping segment, ensuring retrieval of place cells and head direction cells in the loop missed in $D$. 
cells then activates head direction cells that further update the grid cell activity. This process repeats many times during retrieval of the initial nonoverlapping segments. However, when there is an overlapping segment in Figure 7D, the retrieval jumps ahead to the end of the trajectory, missing an entire loop (labeled with $\mathrm{X})$. This framework suffers from the problem of multiple possible head directions being associated with a specific place at different times during an overlapping trajectory. This causes retrieval to fail for sections of the trajectory that contain overlap.

Firing dependent on arc length provides an alternative mechanism for reducing error due to different actions associated with the same location at different times. This involves a hypothetical circuit summarized in Figure 7B with three components: (1) cells dependent on running speed update the entorhinal frequency, (2) entorhinal frequency alters the activity of arc length cells, and (3) arc length cells are associated with specific running speed cells during encoding. In addition, the arc length cells can form associations with specific grid cells and place cells. During retrieval, arc length cells activate the associated running speed that updates the representation of arc length along the trajectory. Thus, retrieval is one-dimensional instead of two-dimensional. This results in sequential activation of arc length cells coding positions at regular intervals along the trajectory (Fig. 7E). This one-dimensional retrieval can then reset the two-dimensional representation in the other circuit by resetting grid cell phase or place cell activity.

As shown in Figure 7C, the arc length code can be associated with spatial location in a trajectory by strengthening connections between arc length cells and entorhinal grid cells (or with head direction cells). As shown in Figure 7F, the arc length representation allows correct retrieval despite overlapping trajectories. Arc length cells fire dependent on the one-dimensional context of the previous segment of the trajectory. This could depend on interactions of multiple oscillations as used in modeling of episodic memory (E.A. Zilli and M.E. Hasselmo, unpubl.). Selective firing of arc length cells can reset grid cell phase and guide selection of one action when many actions are associated with one location. This allows the circuit to retrieve the full trajectory in sequential order (Fig. 7F), despite the overlapping segment. In this manner, arc length cells can enhance the episodic encoding and retrieval of trajectories.

\section{Discussion}

The simulations presented here demonstrate the effectiveness of a model based on oscillatory interference to account for neural responses in continuous spatial alternation (Wood et al. 2000; Lee et al. 2006) and DNMP tasks (Griffin et al. 2007). As shown in Figures $2-4$, the same model effectively simulates the predominant neural responses observed in both tasks. With the addition of reset at reward locations, the model can demonstrate selectivity of firing dependent on reward location (Bower et al. 2005).

This new model accounts for unexpected features of the responses in these tasks. When we designed the DNMP task, we expected neural activity in that task to resemble neural activity seen in continuous spatial alternation, with selective firing dependent on left or right responses, based on our previous model that used strengthening of synapses to encode trajectories (Hasselmo and Eichenbaum 2005). There was some selectivity for left versus right trials in the DNMP task (Griffin et al. 2007), but a larger number of neurons showed specificity for task phase that was difficult to account for in our previous model (Fig. 4). Similarly, the experiment on continuous spatial alternation was guided by hypotheses from the previous model, but demonstrated forward shifting toward goal locations (Lee et al. 2006) that was also difficult to account for in our previous model.
The current model effectively accounts for these seemingly disparate results. As shown in Figure 5, the model responds to regularities in the spatial periodicity of the task. In continuous spatial alternation, there is a constant alternating pattern of left and right responses (L-R-L-R-L-R). Units with regular interference patterns can fire selectively for one or the other trial (Fig. 2). If the spatial periodicity of the units is slightly longer than the task, this causes a gradual task-phase precession in the form of forward shifting of firing location (Fig. 3). In contrast to continuous spatial alternation, in DNMP there is a constant alternating pattern of sample and choice, with randomness of left and right responses (SL-CR-SR-CL-SR-CL-SL-CR). Units with regular interference patterns that cause selective left or right trial firing in spatial alternation will show selective sample or choice firing in DNMP (Fig. 4). These units appear to be functioning as arc length cells, responding to the consistent arc length of the circuit of left versus right in spatial alternation, or the circuit of sample versus choice in DNMP.

This raises the question of how neurons are selected to respond only for this alternating pattern. Neurons show a wide range of frequency selectivity in the entorhinal cortex (Giocomo et al. 2007), and could cause interference with a range of different time scales. One question is why there are not a broad variety of task-phase precession directions observed in the task. Certain frequency differences would cause backward shifting over extended periods, whereas experimentally this was only seen on the first one or two trials on a given day (Mehta et al. 1997; Lee et al. 2006). Perhaps feedback interactions of the hippocampus and entorhinal cortex result in selection of neural activity with a spatial wavelength that matches the spatial wavelength of the behavioral task. The modified version of the model in which firing depends on the latest reward location avoids this problem, as it anchors the spatial dimension of interference to consistent reward locations in the tasks. In the model modified for reward location, forward precession could result from a progressive reduction in oscillation frequency difference or a progressive shift in associations between arc length cells and grid or place cells.

The model using reset of oscillations at reward locations can account for the selective appearance of splitter cells when reward does not occur at the base of the stem (Bower et al. 2005). This modification also accounts for why the forward shift does not move past reward locations (Lee et al. 2006) and firing fields do not shift sides on error trials. In addition, the effect of reward location could also account for the lack of context-dependent firing in a task performed in a Y-maze (Lenck-Santini et al. 2001). In that task, reward was presented at the base of the stem after alternating runs into the left or right arm. Neural firing did not differ in most cells for either outward or inward runs on the stem. In the current model, stopping for reward would reset the oscillations at the base of the stem. The arc length from this location would not differ for either outward or inward runs into the left or right arm, and therefore the model would not show contextdependent responses for either turn direction. Removing the reward at the base of the stem and placing it at the end of the left and right turn arms should cause context-dependent firing in this task. This same framework could also account for the directionality of firing on a linear track, if reward is given at each end of the track.

Many cells fire in a similar location in the stem after left or right turns, but with differential firing rate. This could be called "rate-splitting." This phenomenon can be explained by the model if it is assumed that populations of neurons are reset differently. If a first subset of neurons is reset at the base of the stem, but a second subset is reset at the left reward location, then a neuron receiving input from these two populations would show some firing at the same point in the stem on both trials due to the first 
population, but the firing would be stronger after returning from the left arm because of input from the second population.

This discussion has focused on interactions of entorhinal cortex and hippocampus for context-dependent firing of hippocampal neurons, but similar mechanisms could operate within the entorhinal cortex. This could underlie the evidence for context-dependent firing in the entorhinal cortex (Frank et al. 2000), including splitter cells during continuous alternation (Lipton et al. 2007), and (if reset can be caused by some turns) the change that grid cells undergo when a rat runs in a linear track with many hairpin turns, where firing appears to depend on distance from turning points (Derdikman et al. 2007).

This model uses theta frequency oscillations as a necessary component for the computation of spatial intervals that cause selective firing in the behavioral tasks. This is consistent with data on the behavioral role of theta rhythm oscillations. Lesions that reduce theta rhythm cause behavioral impairments in tasks that require memory for specific prior episodes (Olton et al. 1979; Aggleton and Brown 1999), such as delayed spatial alternation. The amplitude of hippocampal theta oscillations correlates with memory-guided performance (Winson 1978; Givens and Olton 1990; Seager et al. 2002; Griffin et al. 2004), and theta rhythm enhances the synaptic modification that would be necessary to link spatial and context-dependent inputs (Holscher et al. 1997; Hyman et al. 2003). The oscillatory interference model presented here indicates a potential mechanism for the role of hippocampal theta rhythm in memory function.

\section{Predictions of the model}

This model makes predictions about neural firing properties expected in behavioral tasks. For example, several of the cells in continuous spatial alternation do not show context dependence, responding at the same location in the two trial types (nonsplitters) rather than showing different responses (splitter cells). These could be pure place cells, or they could be cells driven at a different period. The splitter cells modeled above have a period of $\sim 20 \mathrm{sec}$ (wavelength of $535 \mathrm{~cm}$ ) between firing fields. A nonsplitter would have a period of $10 \mathrm{sec}$ between firing fields (wavelength of $267 \mathrm{~cm}$ ).

If both types of neurons arise from interference of oscillations, then splitter cells should have firing fields that are twice the size of non-splitter cells, because the longer period associated with a longer wavelength would bring the splitter cell over threshold for twice the spatial length of the non-splitter cell. The cells responding in the DNMP task should have even larger firing fields because the addition of a separate holding area (and the delay period) would increase the spatial length (and temporal duration) of the overall task and cause an increase in the size of firing fields. This seems consistent with the larger firing fields observed in the DNMP task (Griffin et al. 2007) compared to the continuous spatial alternation task (Lee et al. 2006). Conversely, a cell with a period of $5 \mathrm{sec}$ would have two evenly spaced fields on the maze that would be one-quarter the size of a splitter cell firing field.

The continuous spatial alternation task could also have firing fields that skip cycles, for example, appearing once every four trials (e.g., on alternate left trials). Cells that skip cycles should have even larger firing fields. The hippocampus could show firing fields with spacings that fall at quantal integer multiples or factors of the one-dimensional arc length of the alternation task and field sizes that fall at integer multiples or factors of the size of a non-splitter cell field. These cells appear to be constrained to have spatial wavelengths at a scale that fits the wavelength of the behavioral task. The model could generate periodicity resulting in both backward and forward shifting at many different speeds, but the full range of properties does not appear experimentally, suggesting that there is a selection process that favors neurons with quantal sizes that match the scale of the task.

The anatomical position of cell types within the septotemporal axis of the hippocampus should scale with frequency properties of cells along the dorsal-to-ventral axis of the medial entorhinal cortex. More dorsal entorhinal cells have higher intrinsic oscillation frequencies (Giocomo et al. 2007) and project to more septal regions of the hippocampus and should cause responses with higher spatial frequencies (shorter periods). Thus, nonsplitter cells should be located closer to the septum (more rostral) than splitter cells, as splitters have a longer period that is consistent with input from more ventral regions of entorhinal cortex with lower frequencies (longer periods). Cells that skip cycles should appear in more ventral areas. Note that the recordings simulated here were obtained with the middle of the 1-mmdiameter microdrive bundle at $4.0 \mathrm{~mm}$ posterior to bregma and $2.0 \mathrm{~mm}$ lateral to the midline (Griffin et al. 2007). The frequencies observed at individual dorsal-ventral positions in slice recordings (Giocomo et al. 2007) cover the twofold range necessary to underlie both splitters and non-splitters at one recording position (as shown in Fig. 5, task-phase-specific cells would have the same periodicity as splitter cells).

If the cells show coherent forward shifting, then the forward shift for each appearance of a splitter field should be twice the forward shift for each appearance of a non-splitter (so that the overall shift is coherent). In addition, whereas a splitter cell shifts forward into only one reward arm, a non-splitter should shift forward into both reward arms simultaneously, and could be formed by two fields shifting forward simultaneously from both return arms (or after stopping for reward at the stem base).

\section{Relationship to previous models}

The phenomenon of oscillatory interference provides an alternate mechanism for the encoding and retrieval of sequences that differs from previous models and provides more sophisticated timing properties. Most previous hippocampal models encode sequences with Hebbian synaptic modification between activity representing adjacent locations or adjacent items in a sequence, usually by strengthening excitatory recurrent connections in region CA3 (Marr 1971; McNaughton and Morris 1987; Jensen and Lisman 1996a; Levy 1996; Tsodyks et al. 1996; Wallenstein and Hasselmo 1997; Hasselmo 2005b; Howard et al. 2005), or by strengthening excitatory recurrent connections in entorhinal cortex (Hasselmo and Eichenbaum 2005). In contrast, the new model encodes sequences by strengthening associations between neurons representing individual locations (sensory states) and neurons representing characteristics of the action (speed or velocity) guiding the transition to the next state. This model resembles a previous proposal that place cells represent local views, and a cognitive map arises from associations between these local view cells and movements that link these local views (McNaughton and Nadel 1990).

This model has the advantage that it provides an explicit signal for the time course of transitions between states. This solves the problem of regulating the speed of readout of encoded memories. In previous models, the elements of the sequence encoded during behavior would be read out in region CA3 at the time course of AMPA synaptic potentials, resulting in readout much faster than the initially encoded sequence, unless mechanisms for slowing the readout were incorporated in the model (McNaughton and Morris 1987; Jensen and Lisman 1996a; Levy 1996; Tsodyks et al. 1996; Wallenstein and Hasselmo 1997; Hasselmo 2005b; Hasselmo and Eichenbaum 2005; Howard et al. 2005). The mechanisms for regulating retrieval speed included use of slow NMDA-mediated transmission (Jensen and Lisman 
1996b), use of feedback inhibition (Levy 1996; Tsodyks et al. 1996), changes in $\mathrm{GABA}_{\mathrm{B}}$ modulation of synaptic transmission within individual theta cycles (Wallenstein and Hasselmo 1997), and gradual changes in the level of depolarization within theta cycles (Hasselmo and Eichenbaum 2005). In contrast, the readout of trajectories in this model can be regulated at different speeds dependent on the magnitude of frequency changes. This could allow readout in real time as observed during theta rhythm during REM sleep (Louie and Wilson 2001).

The oscillatory interference in this model is primarily driven by sensory input and could therefore contribute to encoding the sensory input. The timing of theta phase precession was previously proposed to underlie encoding of new information (Mehta et al. 2002; Koene et al. 2003; Sato and Yamaguchi 2003; Yamaguchi 2003; Jensen and Lisman 2005), based on formation of associations between states. This contrasts with the current model that focuses on associations between states and the actions (speed or velocity) that mediate transitions between states. Units representing action could hold graded levels of activity as demonstrated experimentally in single entorhinal neurons (Egorov et al. 2002; Fransén et al. 2006), thereby holding a continuous signal coding speed and head direction during transition between locations (and potentially computing mean velocity over adjacent states). Formation of associations between states and actions allows this model to perform retrieval in the same time scale as encoding, which would allow precession during retrieval of a sequence similar to models in which precession is due to retrieval of previously encoded associations (Jensen and Lisman 1996a; Tsodyks et al. 1996; Hasselmo 2005b; Hasselmo and Eichenbaum 2005).

In previous models, the context-dependent retrieval of sequences was usually obtained with a context representation in hippocampal region CA3. The representation of context could result from activity spreading across previously modified synaptic connections within region CA3 (Levy 1996; Wallenstein and Hasselmo 1997), or could result from persistent spiking representing context in entorhinal cortex layer II and spreading through region CA3 (Hasselmo and Eichenbaum 2005). Experiments were performed to test predictions about the theta phase of neural firing from the latter model, but instead generated the observation of forward shifting. Forward shifting was not easy to simulate in the associative retrieval model, as strengthening of excitatory synaptic connections usually causes a backward shift of firing location in these types of models (Blum and Abbott 1996; Mehta et al. 1997). Similarly, it was difficult to simulate the task-phase selectivity observed in DNMP using models based on strengthening of synapses to perform associative retrieval. In contrast to both associative retrieval and working memory models, the model based on interference of oscillations can account for the different properties of the data. These simulations do not directly address how the retrieval of the trajectory can be used for performance of the task. However, other simulations have shown that performance can be guided by retrieval of the most recently performed trajectory in the task (Hasselmo and Eichenbaum 2005; Zilli and Hasselmo 2007).

\section{Relationship of models to data on hippocampal subregions}

Different theories have proposed different functions for hippocampal subregions. Here the three theories are referred to as (1) the standard model, (2) the context model, and (3) the oscillatory interference model. These models share certain features, including the role of entorhinal cortex in providing both input and output to the system, and the role of the dentate gyrus in performing pattern separation, forming sparse representations of sensory input to enhance efficiency of encoding.
The models differ in their proposed function for regions CA3 and CA1. In the standard model, the excitatory recurrent connections in CA3 form the basis for associations of an item with itself and with other items or with context (McNaughton and Morris 1987; Treves and Rolls 1994; Hasselmo et al. 1995). A separate comparator stage in region CA1 can then gate encoding or retrieval of the associations in CA3 (Hasselmo and Schnell 1994; Treves and Rolls 1994; Hasselmo and Wyble 1997). In the context model, region CA3 provides contextual input that allows disambiguation of one sequence of activity from another sequence either within region CA3 (Levy 1996), or via interaction in region CA1 with sequence retrieval from entorhinal cortex (Hasselmo and Eichenbaum 2005).

As noted in Materials and Methods, the model of episodic memory presented here forms associations between states and the intervening actions, using oscillatory interference to time the intervals of actions between sequential states. As in previous models, the dentate gyrus and region CA3 form distinct state representations of location, while region CA1 responds on the basis of action inputs mediating relative timing or arc length between states. Each new state activates a new action representation consisting of persistent firing in entorhinal cortex. This persistent spiking drives oscillatory interference to time the interval to the next state scaled by retrieved speed or velocity.

Several experimental studies support the new model, as well as aspects of the previous models. Studies support a role for region CA3 in computing spatial context, whereas CA1 appears important for storing the relative order of events. Selective lesions of region CA3 impair the capability of a rat to show a novelty response of increased sniffing when a familiar object is moved to a new location (Lee et al. 2005a). This impairment could result from loss of spatial representations in CA3 due to direct input from entorhinal cortex layer II (Fyhn et al. 2007). These spatial representations could influence novelty responses via output connections with the septum (Hasselmo 2005a) rather than output via region CA1, as CA1 lesions cause less of an effect on recognition behavior (Lee et al. 2005a).

Neuronal recordings support a greater role for CA3 in coding spatial context, whereas region CA1 could code relative order. Place cells in region CA3 shift in a coherent manner with cues shifted around a circular track in a single room (Lee et al. 2004), in contrast to more heterogeneous responses of region CA1 neurons to these changes (suggesting that CA1 may be computing intervals between stimuli rather than spatial context). When similar local cues are encountered in a completely different location, place cells in CA3 show remapping indicative of a response dependent on the spatial location (Leutgeb et al. 2004). This remapping appears to arise from shifts in the relative orientation of grid cells in entorhinal cortex (Fyhn et al. 2007). The sensitivity to the context of spatial location appears to result from a nonlinear response to environment similarity, in which CA3 neural activity shows completion effects for similar spatial contexts, whereas more distinct contexts result in greater differentiation of neuronal response in region CA3 (Guzowski et al. 2004; Vazdarjanova and Guzowski 2004).

Whereas region CA3 appears important for coding spatial context, region CA1 may be more important for retrieval of the temporal order of items or events. Rats with full hippocampal lesions show impairments in retrieval on the basis of the temporal order of items (Fortin et al. 2002; Kesner et al. 2002), and selective CA1 lesions appear to cause greater impairments than CA3 lesions for memory of the temporal order of items (Lee et al. 2005b; Rolls and Kesner 2006; Hoge and Kesner 2007). Region CA1 lesions but not CA3 lesions impair the ability of rats to perform a task in which an odor must be compared with the same or different odor after a 10-sec delay, suggesting a role for CA1 in 
context-dependent timing of intervals (Kesner et al. 2005; Manns and Eichenbaum 2005). Lower-frequency oscillations in more ventral entorhinal neurons could be important for timing of longer temporal intervals, as suggested by greater impairments of trace fear conditioning by ventral hippocampal lesions (Rogers et al. 2006; Yoon and Otto 2007). In summary, the experimental data support the notion of a separate neuronal population computing one-dimensional arc length in addition to place cells responding to spatial location in two dimensions. Thus, this supports hippocampal mechanisms for encoding memories using additional representations beyond two-dimensional space (Eichenbaum et al. 1999).

\section{Materials and Methods}

\section{Movement of virtual rat}

To demonstrate the firing properties of the model during realistic behavior, a simulated rat was guided through movements in space corresponding to each of two behavioral tasks used for collecting previously published data-continuous spatial alternation (Wood et al. 2000; Lee et al. 2006) and DNMP (Griffin et al. 2007). Both experimental tasks were performed on the same Tmaze apparatus with a stem length of $116 \mathrm{~cm}$ and reward arm lengths of $53.5 \mathrm{~cm}$ each (totaling $107 \mathrm{~cm}$ ). The T-maze had return arms running from the reward site at the end of each reward arm back to the base of the stem.

The first behavioral task was the continuous spatial alternation task (Wood et al. 2000; Lee et al. 2006). In this task, the rat must alternate its response at the end of the stem to go right or left on alternate trials, as shown in Figure 1A. In these simulations, the virtual rat was timed to run the task at about the same speed as a real rat, with a 10-sec period between one reward site and another reward site, giving an average running speed of $\sim 26$ $\mathrm{cm} / \mathrm{sec}$. In all simulations, the speed of the rat was made to change randomly around the mean. This ensured that the trajectory of the simulated rat would appear in slightly different locations on each trial, allowing a better match to the variation in rat trajectory observed in the experimental data. Note that the simulation automatically generated correct spatial behavior of the rat, to replicate the performance of the fully trained rat in the experiment when it was showing few errors. In future models, the neural activity could be used to guide behavior, as in other simulations in which episodic retrieval (Hasselmo and Eichenbaum 2005) and/or working memory (E.A. Zilli and M.E. Hasselmo, unpubl.) guided the behavioral choice of the virtual rat.

The second behavioral task was DNMP (Griffin et al. 2007), as shown in Figure 1B. In this task, sequential pairs of trials included one sample trial followed by a separate choice trial. On the sample trial, the simulated rat runs up the stem of the maze and is forced to go either left or right by a blockade at the start of the reward arm. On the subsequent choice trial, the simulated rat runs up the stem of the maze and goes to the opposite reward arm. The simulation replicated correct performance in the task, which consisted of a random series of sample trials, with each sample followed by the correct response to the opposite side on the choice trial. The same variation of speed was used in this task. Note that the simulation does not directly simulate the waiting platform used during the intertrial interval before the sample trial in DNMP.

\section{Simulation of interfering oscillatory activity}

The spiking activity in this model arises from the interaction of two oscillatory inputs. This corresponds to a simplified version of a model developed to simulate entorhinal grid cells (Burgess et al. 2007), and is closely related to models of theta phase precession using interference between oscillations (O'Keefe and Recce 1993; Bose et al. 2000; Lengyel et al. 2003; O'Keefe and Burgess 2005).

In this model, the oscillations represent the network theta rhythm in the hippocampus (at $\sim 6 \mathrm{~Hz}$ ) interacting with the theta rhythmic input from neurons in the entorhinal cortex that have about the same frequency but are modulated by running speed.
Extensive data show theta rhythm oscillations in both regions and modulation by running speed. Theta rhythm oscillations have been studied for many decades in the hippocampus (Green and Arduini 1954; Fox et al. 1986; Brankack et al. 1993; Csicsvari et al. 1999; Buzsaki 2002; Hasselmo 2005b; O'Keefe and Burgess 2005). Hippocampal theta rhythm is paced by rhythmic input from the medial septum, regulating inhibition in stratum pyramidale (Stewart and Fox 1990; Toth et al. 1997; Buzsaki 2002), and is also associated with rhythmic sinks and sources in stratum radiatum and stratum lacunosum-moleculare (Brankack et al. 1993; Buzsaki 2002; Hasselmo 2005b). Speed modulation of the frequency of entorhinal input could directly influence the frequency of synaptic input in stratum lacunosum-moleculare. Alternately, membrane potential oscillations mediated by the $\mathrm{H}$ current in distal dendrites could account for the strong current sinks in stratum lacunosum-moleculare (Brankack et al. 1993) and might be regulated in frequency or phase by entorhinal neurons that do not necessarily fire at the same phase (Stewart et al. 1992).

Theta rhythm oscillations have also been shown extensively in the entorhinal cortex with both field potential and unit recording (Mitchell and Ranck 1980; Mitchell et al. 1982; Alonso and Garcia-Austt 1987a,b; Stewart et al. 1992; Quirk et al. 1992). Theta rhythmic firing in entorhinal cortex may partly result from contributions of intrinsic membrane potential oscillations (Acker et al. 2003; Fransén et al. 2004; Giocomo et al. 2007) or theta rhythmic properties of persistent spiking activity (Tahvildari et al. 2007).

The model is supported by data showing that running speed influences the frequency of theta rhythm (Whishaw and Vanderwolf 1973; Rivas et al. 1996; Slawinska and Kasicki 1998; Maurer et al. 2005). The modulation of entorhinal frequency (or distal dendritic frequency) by running speed $s$ should alter the frequency of current sinks in stratum lacunosum-moleculare of region CA1 (Brankack et al. 1993). Thus, the model correlates with the previously observed modulation of the frequency of hippocampal theta rhythm by different running speeds (Whishaw and Vanderwolf 1973; Rivas et al. 1996; Slawinska and Kasicki 1998; Maurer et al. 2005). A recent measurement of the slope of the change in frequency with running speed in the hippocampus showed a mean slope of $0.0057 \mathrm{~Hz} \cdot \mathrm{sec} / \mathrm{cm}$ for dorsal hippocampus and $0.0098 \mathrm{~Hz} \cdot \mathrm{sec} / \mathrm{cm}$ for middle regions of hippocampus (Maurer et al. 2005). This is consistent with the magnitude of speed modulation used in previous models of grid cells (Hasselmo et al. 2007).

\section{Equations of the model}

The interaction of oscillations used for coding arc length is expressed in the following equation:

$$
o(t)=\Theta\{\cos [f 2 \pi t]+\cos [(f+f B s(t)) 2 \pi t+\varphi]\}
$$

where $o(t)$ represents the firing of hippocampal pyramidal cells over time. The frequency of hippocampal theta is represented with $f$. The frequency of theta rhythmic input from entorhinal cortex is represented with $f_{E}=f+f B s(t)$. When the magnitude of these interacting oscillations crosses a threshold, they will generate a spiking response. This output function is represented by the Heaviside step function $\Theta[$. . . The initial oscillation phase is set by $\varphi$, which determines the firing location along the trajectory. The running speed of the rat that regulates entorhinal frequency is represented by the function $s(t)$. The constant $B$ scales the change in frequency associated with running speed. Speed modulation of neural activity has been shown for subcortical structures including the habenula and medial mammillary nucleus (Sharp and Turner-Williams 2005; Sharp et al. 2006). Clear speed modulation of neural activity has also been shown for axons in the hippocampus (O'Keefe et al. 1998), suggesting that speed-dependent activity is available to hippocampal structures. Speed modulation has also been shown for neurons in areas with head direction cells such as the postsubiculum (Taube et al. 1990; Sharp 1996) and in deep layers of entorhinal cortex (Sargolini et al. 2006), but the simulation presented here focuses 
on speed modulation independent of head direction. The model could also function if running speed altered hippocampal theta rhythm frequency (Whishaw and Vanderwolf 1973; Rivas et al. 1996; Slawinska and Kasicki 1998; Maurer et al. 2005), instead of the theta rhythmic frequency of entorhinal input.

Let us consider the interference of the two oscillations in Equation 1. They will show a beat pattern due to consecutive periods of constructive and destructive interference that result in increases and decreases in the envelope of the oscillation (see Fig. $5 \mathrm{~A}$ ). The frequency of this envelope, or beat frequency $f_{b}$, is equal to the difference between the two frequencies $f_{E}-f=f B s(t)$. The phase of this interference will change according to

$$
\Delta \varphi=f B 2 \pi s(t) \Delta t
$$

where $\varphi$ is the phase of the oscillation envelope that determines when the interference oscillation crosses threshold. The speed of the rat can be computed from its movements in the $x$ and $y$ dimensions with

$$
s(t)=\sqrt{(d x / d t)^{2}+(d y / d t)^{2}}
$$

Putting the equation for speed into the equation for phase (Equation 2) and computing the integral of Equation 2 yields

$$
\varphi=f B 2 \pi \int_{a}^{b} \sqrt{(d x / d t)^{2}+(d y / d t)^{2}} d t
$$

The integral in this equation is the general mathematical solution for computing the arc length of a trajectory (irregular arc segment) through two-dimensional space. Thus, the phase of the oscillatory interference is proportional to the arc length that the rat has traveled on a given trajectory since the start of the computation (scaled by the beat frequency $f B$ ).

If we take away the speed modulation, then the phase depends on the duration of time $t$ alone:

$$
\varphi=f B 2 \pi t
$$

If instead of taking away the speed modulation, we add modulation by head direction, we obtain

$$
\Delta \varphi=f B 2 \pi \sqrt{(d x / d t)^{2}+(d y / d t)^{2}} \cos [\arctan (d y / d x)]=f_{b} 2 \pi \Delta x
$$

This gives changes in phase dependent on the allocentric Cartesian coordinate $x$ (relative to the head direction). The version incorporating head direction forms the basis for the previous model of formation of grid cells (Burgess et al. 2005, 2007) further analyzed in Hasselmo et al. (2007). That model combines input from multiple head directions to generate grid patterns and provide a mechanism for path integration.

The above equations show that the phase of interference has the capacity to code for the essential components of episodic memory function-when and where. They provide a means for encoding a continuous trajectory through space and time. Input from lateral entorhinal cortex could provide the additional element of "what" that can be linked to a specific position in the continuous representation of "where" and "when."

\section{Analysis of firing field stability versus forward shifting}

This section provides quantitative details of the simulation of spatial periodicity in the behavioral tasks. For the simulations presented in Figures 2-4, the firing properties depend on the spatial periodicity of the model relative to the overall spatial length of the task. In the simulations of continuous spatial alternation shown in Figures 2 and 3, the hippocampal frequency is $f=6 \mathrm{~Hz}$, and the entorhinal frequency takes the form $f+f B^{*} s$, where $f=6 \mathrm{~Hz}$ and $f B=1.84 \times 10^{-3}$. Thus, at a running speed of $s=26 \mathrm{~cm} / \mathrm{sec}$, the average frequency of entorhinal input is $6.0478 \mathrm{~Hz}$. Note that this is just the average, as this frequency will vary as the speed of the rat varies randomly around the mean. The difference in frequency between hippocampus and entorhinal cortical input of $0.0478 \mathrm{~Hz}$ gives an approximate period $(T=1 / f)$ between spiking in firing fields of $20.9 \mathrm{sec}$, and an ap- proximate arc length of $543.4 \mathrm{~cm}$. The length of the full circuit of the alternation task is $\sim 536 \mathrm{~cm}$ (this includes the length of the L to $\mathrm{R}$ trial plus the $\mathrm{R}$ to $\mathrm{L}$ trial). Thus, the forward shifting is $\sim 7 \mathrm{~cm}$ per trial. The initial location of firing was determined by setting the phase $\varphi$ at $\pi / 2.8$ radians in Figure $2 \mathrm{~B} 1$ and at $\pi+\pi / 2.8$ radians in Figure 2B2. For simulations of DNMP (DNMP) in Figure 4, $f B$ was set to a slightly higher value of $1.85 \times 10^{-3}$, giving a slightly slower forward shift. The phase was set at zero in Figure $4 \mathrm{~B}$ and $\pi$ radians $\left(180^{\circ}\right)$ in Figure $4 \mathrm{D}$. The firing location will not shift forward if $f B=1.87 \times 10^{-3}$, which would give an average entorhinal frequency of 6.0486 . Note that doubling the scaling $f B$ to $3.74 \times 10^{-3}$ would cause the period to be $\sim 10 \mathrm{sec}$, resulting in firing on both the $\mathrm{L}$ to $\mathrm{R}$ and $\mathrm{R}$ to $\mathrm{L}$ circuit. This will appear as spatially localized firing (non-splitters), with cells firing at the same location during both $\mathrm{L}$ to $\mathrm{R}$ and $\mathrm{R}$ to $\mathrm{L}$ trials. This indicates that different magnitudes of the speed modulation of frequency will give different patterns of firing in the task.

Note that there is less dependence on the long-term periodicity in the version of the model where persistent firing is reset after stopping at a reward location. Simulations in Figure 6 use a modified version of the model in which entorhinal input arises from different populations of entorhinal neurons that are activated at specific reward locations. For example, when the rat reaches the reward location in the left arm, previous persistent firing stops, and thereby previous oscillations are terminated. When the rat starts again from this position, persistent firing in a different set of neurons is activated, thereby starting oscillations in a separate set of neurons representing the left arm reward location. When the rat then reaches the reward location in the right arm, the persistent firing associated with the left arm reward location stops, and those oscillations are terminated. When it starts again from the right arm reward location, persistent firing and oscillations are induced in the set of neurons representing the right arm reward location. Thus, the persistent spiking buffer is different at left versus right reward locations, and thereby activates different context-dependent neurons (Fig. 6A). For simulation of a reward location at the base of the stem, the persistent spiking buffer is activated for the same location at the base of the stem for each trial type, resulting in less dependence on left versus right responses (Fig. 6B).

\section{Model of episodic memory}

The model of episodic memory involves two different loops (Fig. 7). As shown in Figure 7A, the first loop retrieves trajectories via three stages: (1) head direction cells update grid cells, (2) grid cells update place cells, and (3) place cells activate associated head direction activity. The first stage of the model uses input from head direction cells to create grid cell firing responses. This has been effectively described in a previously developed model (Burgess et al. 2005, 2007) as follows:

$$
g(t)=\prod_{H D} \Theta\left\{\cos [f 2 \pi t]+\cos \left[\left(f+f B s \cos \left(\phi-\phi_{H D}\right)\right) 2 \pi t+\varphi\right]\right\}
$$

This model effectively uses input from three HD cells to create grids with spacing dependent on frequency $f$. This model is supported by physiological data on membrane potential oscillations in entorhinal cortex (Giocomo et al. 2007; Hasselmo et al. 2007). The phase vector of the grid cells sums (or integrates) the speed-modulated head direction cell input $H(t)$ determined by spatial location.

$$
g(t+\Delta t)=\prod_{H D}\left[\cos \left(f 2 \pi \sum_{t} H(t) \Delta t+\varphi\right)\right]
$$

The time interval $\Delta t$ was $20 \mathrm{msec}$ in the simulations shown in Figure 7.

The second stage involves grid cell effects on place cells. This stage could be done in various ways, as other studies have explicitly simulated how place cells could arise from grid cells (Rolls et al. 2006; Solstad et al. 2006; E.A. Zilli and M.E. Hasselmo, unpubl.), but research has not converged on a single model. In general, one could consider the place cell representation $p(t)$ in 
region CA3 arising from the grid cell representation $g(t)$ in entorhinal cortex layer II via synaptic connectivity $W_{P G}$ :

$$
p(t)=W_{P G g} g(t)
$$

For a given environment, the first two stages could be fixed (although this is not necessary for the model to function). In the simulations presented here, individual place representations are formed at intervals along the trajectory during encoding. During retrieval, the phase of entorhinal grid cell activity is updated by head direction input, and the place cell closest to the location represented by this phase is activated.

The encoding of a trajectory could be performed entirely by the third stage of the circuit, involving connections between place cells and head direction cells. Initial encoding of a trajectory could involve strengthening a set of synapses $W_{H P}$ between place cells coding each location and speed-modulated head direction cells coding the associated action. Thus, the strengthening of synapses could take the form:

$$
W_{H P}=\sum_{t} H(t) p(t)^{T}
$$

Subsequently, when it is useful to retrieve the trajectory at a later time, the head direction activity (action) after each interval $\Delta t$ can be retrieved based on the previous head direction activity updating the phase of grid cell activity $g$ as follows:

$$
H(t+\Delta t)=W_{H P} W_{P G} g(H(t) \Delta t)
$$

For a discrete time representation, one set of head direction responses (coding a velocity vector corresponding to rat action) would need to maintain activity until a new place cell representation causes retrieval of the next head direction response. This would require graded levels of persistent firing that have been shown in deep layers of entorhinal cortex (Egorov et al. 2002; Fransén et al. 2006). This suggests that deep layer entorhinal grid cells that also respond to head direction (Sargolini et al. 2006) may be involved in encoding and retrieval of trajectories.

Trajectory retrieval can be greatly enhanced by combining the mechanism based on place cells $p(t)$ with the mechanisms of context-dependent firing $o(t)$ dependent on arc length in Equation 1. The circuit using arc length cells is summarized in Figure $7 \mathrm{~B}$. Note that the simulations of arc length in Figure 7 use $f B=1.54 \times 10^{-2}$, which results in more frequent suprathreshold activity along the trajectory. Each new arc length cell $a(t)$ could be activated by the preceding cell plus spiking from oscillatory interference.

Analogous to the connection between place cells and head direction cells $\left(W_{H P}\right)$, each arc length cell $a(t)$ can be linked with the associated speed $s(t)$ at each position along the trajectory, as shown in Figure 7B.

$$
W_{S O}=\sum_{t} s(t) a(t)^{T}
$$

This enhances retrieval because the mechanism for activation of the context-dependent arc length cells does not depend on retrieval activating an accurate vector of head direction activity at each step. Instead, it only requires that retrieval activate a representation of speed. Thus, retrieval is one-dimensional instead of two-dimensional:

$$
s(t)=W_{S O} a(s(t))
$$

As shown in Figure 7C, the arc length code can be associated with spatial location in a trajectory by strengthening connections between arc length cells $a(t)$ in CA1 and the grid cells firing at a specific phase, so that arc length cells can reset the representation of location by phase. During retrieval, the combined model uses interaction of the circuit in Figure 7A and the circuit in Figure 7B. In Figure 7A, grid cell phase activates place cells, which update head direction activity to drive grid cell phase to the next place cell. Simultaneously, entorhinal persistent spiking cells update arc length cells to drive speed cells to activate the appropriate level of persistent spiking (Fig. 7B). In Figure 7C, the arc length cells also drive grid cells to have the appropriate phase. If a single place cell is associated with multiple head directions because of overlapping trajectories, the trajectory retrieval can be disambiguated by arc length cells that activate the correct grid cell phase (or head direction cells). This allows retrieval of the complete trajectory as shown in Figure 7F. The retrieval process can be accelerated or decelerated via modulation of the frequency of entorhinal oscillations during persistent firing.

\section{Acknowledgments}

This research was supported by Silvio O. Conte Center grant NIMH MH71702, NIMH R01 60013, NIMH MH61492, NIMH MH60450, NSF SLC SBE 0354378, and NIDA DA16454 (part of the CRCNS program). The simulations presented in this paper focus on published experimental data gathered in my laboratory by Inah Lee (now at the University of Iowa) and Amy Griffin (now at the University of Delaware) in collaboration with Howard Eichenbaum.

\section{References}

Acker, C.D., Kopell, N., and White, J.A. 2003. Synchronization of strongly coupled excitatory neurons: Relating network behavior to biophysics. J. Comput. Neurosci. 15: 71-90.

Aggleton, J.P. and Brown, M.W. 1999. Episodic memory, amnesia, and the hippocampal-anterior thalamic axis. Behav. Brain Sci. 22: $425-489$.

Alonso, A. and Garcia-Austt, E. 1987a. Neuronal sources of theta rhythm in the entorhinal cortex of the rat. I. Laminar distribution of theta field potentials. Exp. Brain Res. 67: 493-501.

Alonso, A. and Garcia-Austt, E. 1987b. Neuronal sources of theta rhythm in the entorhinal cortex of the rat. II. Phase relations between unit discharges and theta field potentials. Exp. Brain Res. 67: 502-509.

Alonso, A. and Klink, R. 1993. Differential electroresponsiveness of stellate and pyramidal-like cells of medial entorhinal cortex layer II. J. Neurophysiol. 70: 128-143.

Alonso, A. and Llinas, R.R. 1989. Subthreshold Na-dependent theta-like rhythmicity in stellate cells of entorhinal cortex layer II. Nature 342: 175-177.

Blum, K.I. and Abbott, L.F. 1996. A model of spatial map formation in the hippocampus of the rat. Neural Comput. 8: 85-93.

Bose, A. and Recce, M. 2001. Phase precession and phase-locking of hippocampal pyramidal cells. Hippocampus 11: 204-215.

Bose, A., Booth, V., and Recce, M. 2000. A temporal mechanism for generating the phase precession of hippocampal place cells. $J$. Comput. Neurosci. 9: 5-30.

Bower, M.R., Euston, D.R., and McNaughton, B.L. 2005 Sequential-context-dependent hippocampal activity is not necessary to learn sequences with repeated elements. J. Neurosci. 25: $1313-1323$.

Brankack, J., Stewart, M., and Fox, S.E. 1993. Current source density analysis of the hippocampal theta rhythm: Associated sustained potentials and candidate synaptic generators. Brain Res. 615: $310-327$

Burgess, N., Barry, C., Jeffery, K.J., and O'Keefe, J. 2005. A grid and place cell model of path integration utilizing phase precession versus theta. Computational Cognitive Neuroscience Meeting, Washington, DC.

Burgess, N., Barry, C., and O'Keefe, J. 2007. An oscillatory interference model of grid cell firing. Hippocampus 17: 801-812.

Buzsaki, G. 2002. Theta oscillations in the hippocampus. Neuron 33: $325-340$.

Buzsaki, G., Leung, L.W., and Vanderwolf, C.H. 1983. Cellular bases of hippocampal EEG in the behaving rat. Brain Res. 287: 139-171.

Csicsvari, J., Hirase, H., Czurko, A., Mamiya, A., and Buzsaki, G. 1999. Oscillatory coupling of hippocampal pyramidal cells and interneurons in the behaving rat. J. Neurosci. 19: 274-287.

Derdikman, D., Fyhn, M., Hafting, T., Moser, M.-B., and Moser, E.I. 2007. Breaking up the entorhinal grid in a hairpin maze. Soc. Neurosci. Abstr. 33: 68.10.

Dickson, C.T., Magistretti, J., Shalinsky, M.H., Fransén, E., Hasselmo, M.E., and Alonso, A. 2000. Properties and role of $I_{\mathrm{h}}$ in the pacing of subthreshold oscillations in entorhinal cortex layer II neurons. $J$. Neurophysiol. 83: 2562-2579.

Egorov, A.V., Hamam, B.N., Fransén, E., Hasselmo, M.E., and Alonso, A.A. 2002. Graded persistent activity in entorhinal cortex neurons. Nature 420: $173-178$.

Eichenbaum, H., Dudchenko, P., Wood, E., Shapiro, M., and Tanila, H. 
1999. The hippocampus, memory, and place cells: Is it spatial memory or a memory space? Neuron 23: 209-226.

Fortin, N.J., Agster, K.L., and Eichenbaum, H.B. 2002. Critical role of the hippocampus in memory for sequences of events. Nat. Neurosci. 5: $458-462$.

Fox, S.E., Wolfson, S., and Ranck Jr., J.B. 1986. Hippocampal theta rhythm and the firing of neurons in walking and urethane anesthetized rats. Brain Res. 62: 495-508.

Frank, L.M., Brown, E.N., and Wilson, M. 2000. Trajectory encoding in the hippocampus and entorhinal cortex. Neuron 27: 169-178.

Fransén, E., Alonso, A.A., and Hasselmo, M.E. 2002. Simulations of the role of the muscarinic-activated calcium-sensitive nonspecific cation current INCM in entorhinal neuronal activity during delayed matching tasks. J. Neurosci. 22: 1081-1097.

Fransén, E., Alonso, A.A., Dickson, C.T., Magistretti, J., and Hasselmo, M.E. 2004. Ionic mechanisms in the generation of subthreshold oscillations and action potential clustering in entorhinal layer II stellate neurons. Hippocampus 14: 368-384.

Fransén, E., Tahvildari, B., Egorov, A.V., Hasselmo, M.E., and Alonso, A.A. 2006. Mechanism of graded persistent cellular activity of entorhinal cortex layer v neurons. Neuron 49: 735-746.

Fyhn, M., Hafting, T., Treves, A., Moser, M.B., and Moser, E.I. 2007. Hippocampal remapping and grid realignment in entorhinal cortex. Nature 446: 190-194.

Geisler, C., Robbe, D., Zugaro, M., Sirota, A., and Buzsaki, G. 2007. Hippocampal place cell assemblies are speed-controlled oscillators. Proc. Natl. Acad. Sci. 104: 8149-8154.

Giocomo, L.M., Zilli, E.A., Fransén, E., and Hasselmo, M.E. 2007. Temporal frequency of subthreshold oscillations scales with entorhinal grid cell field spacing. Science 315: 1719-1722.

Givens, B.S. and Olton, D.S. 1990. Cholinergic and GABAergic modulation of the medial septal area: Effect on working memory. Behav. Neurosci. 104: 849-855.

Green, J.D. and Arduini, A.A. 1954. Hippocampal electrical activity and arousal. J. Neurophysiol. 17: 533-557.

Griffin, A.L., Asaka, Y., Darling, R.D., and Berry, S.D. 2004. Theta-contingent trial presentation accelerates learning rate and enhances hippocampal plasticity during trace eyeblink conditioning. Behav. Neurosci. 118: 403-411.

Griffin, A.L., Eichenbaum, H., and Hasselmo, M.E. 2007. Spatial representations of hippocampal CA1 neurons are modulated by behavioral context in a hippocampus-dependent memory task. $J$. Neurosci. 27: 2416-2423.

Guzowski, J.F., Knierim, J.J., and Moser, E.I. 2004. Ensemble dynamics of hippocampal regions CA3 and CA1. Neuron 44: 581-584.

Hafting, T., Fyhn, M., Molden, S., Moser, M.B., and Moser, E.I. 2005. Microstructure of a spatial map in the entorhinal cortex. Nature 436: $801-806$

Hasselmo, M.E. 2005a. The role of hippocampal regions CA3 and CA1 in matching entorhinal input with retrieval of associations between objects and context: Theoretical comment on Lee et al. (2005). Behav. Neurosci. 119: 342-345.

Hasselmo, M.E. 2005b. What is the function of hippocampal theta rhythm?-Linking behavioral data to phasic properties of field potential and unit recording data. Hippocampus 15: 936-949.

Hasselmo, M.E. and Eichenbaum, H. 2005. Hippocampal mechanisms for the context-dependent retrieval of episodes. Neural Netw. 18: $1172-1190$.

Hasselmo, M.E. and Schnell, E. 1994. Laminar selectivity of the cholinergic suppression of synaptic transmission in rat hippocampal region CA1: Computational modeling and brain slice physiology. $J$. Neurosci. 14: 3898-3914.

Hasselmo, M.E. and Wyble, B.P. 1997. Free recall and recognition in a network model of the hippocampus: Simulating effects of scopolamine on human memory function. Behav. Brain Res. 89: $1-34$

Hasselmo, M.E., Schnell, E., and Barkai, E. 1995. Dynamics of learning and recall at excitatory recurrent synapses and cholinergic modulation in rat hippocampal region CA3. J. Neurosci. 15: $5249-5262$.

Hasselmo, M.E., Giocomo, L.M., and Zilli, E.A. 2007. Grid cell firing may arise from interference of theta frequency membrane potentia oscillations in single neurons. Hippocampus (in press).

Hoge, J. and Kesner, R.P. 2007. Role of CA3 and CA1 subregions of the dorsal hippocampus on temporal processing of objects. Neurobiol. Learn. Mem. 88: 225-231.

Holscher, C., Anwyl, R., and Rowan, M.J. 1997. Stimulation on the positive phase of hippocampal theta rhythm induces long-term potentiation that can be depotentiated by stimulation on the negative phase in area CA1 in vivo. J. Neurosci. 17: 6470-6477.

Howard, M.W., Fotedar, M.S., Datey, A.V., and Hasselmo, M.E. 2005. The temporal context model in spatial navigation and relational learning: Toward a common explanation of medial temporal lobe function across domains. Psychol. Rev. 112: 75-116.

Hyman, J.M., Wyble, B.P., Goyal, V., Rossi, C.A., and Hasselmo, M. 2003. Stimulation in hippocampal region CA1 in behaving rats yields LTP when delivered to the peak of theta and LTD when delivered to the trough. J. Neurosci. 23: 11725-11731.

Jensen, O. and Lisman, J.E. 1996a. Hippocampal CA3 region predicts memory sequences: Accounting for the phase precession of place cells. Learn. Mem. 3: 279-287.

Jensen, O. and Lisman, J.E. 1996b. Theta/gamma networks with slow NMDA channels learn sequences and encode episodic memory: Role of NMDA channels in recall. Learn. Mem. 3: 264-278.

Jensen, O. and Lisman, J.E. 2005. Hippocampal sequence-encoding driven by a cortical multi-item working memory buffer. Trends Neurosci. 28: 67-72.

Kesner, R.P., Gilbert, P.E., and Barua, L.A. 2002. The role of the hippocampus in memory for the temporal order of a sequence of odors. Behav. Neurosci. 116: 286-290.

Kesner, R.P., Hunsaker, M.R., and Gilbert, P.E. 2005. The role of CA1 in the acquisition of an object-trace-odor paired associate task. Behav. Neurosci. 119: 781-786.

Klink, R. and Alonso, A. 1997a. Ionic mechanisms of muscarinic depolarization in entorhinal cortex layer II neurons. J. Neurophysiol. 77: $1829-1843$

Klink, R. and Alonso, A. 1997b. Muscarinic modulation of the oscillatory and repetitive firing properties of entorhinal cortex layer II neurons. J. Neurophysiol. 77: 1813-1828.

Koene, R.A., Gorchetchnikov, A., Cannon, R.C., and Hasselmo, M.E. 2003. Modeling goal-directed spatial navigation in the rat based on physiological data from the hippocampal formation. Neural Netw. 16: $577-584$.

Lee, I., Yoganarasimha, D., Rao, G., and Knierim, J.J. 2004. Comparison of population coherence of place cells in hippocampal subfields CA1 and CA3. Nature 430: 456-459.

Lee, I., Hunsaker, M.R., and Kesner, R.P. 2005a. The role of hippocampal subregions in detecting spatial novelty. Behav. Neurosci. 119: $145-153$

Lee, I., Jerman, T.S., and Kesner, R.P. 2005b. Disruption of delayed memory for a sequence of spatial locations following CA1- or CA3-lesions of the dorsal hippocampus. Neurobiol. Learn. Mem. 84: $138-147$.

Lee, I., Griffin, A.L., Zilli, E.A., Eichenbaum, H., and Hasselmo, M.E. 2006. Gradual translocation of spatial correlates of neuronal firing in the hippocampus toward prospective reward locations. Neuron 51: 639-650.

Lenck-Santini, P.P., Save, E, and Poucet, B. 2001. Place-cell firing does not depend on the direction of turn in a Y-maze alternation task. Eur. J. Neurosci. 13: 1055-1058.

Lengyel, M., Szatmary, Z., and Erdi, P. 2003. Dynamically detuned oscillations account for the coupled rate and temporal code of place cell firing. Hippocampus 13: 700-714.

Leutgeb, S., Leutgeb, J.K., Treves, A., Moser, M.B., and Moser, E.I. 2004 Distinct ensemble codes in hippocampal areas CA3 and CA1. Science 305: $1295-1298$.

Levy, W.B. 1996. A sequence predicting CA3 is a flexible associator that learns and uses context to solve hippocampal-like tasks. Hippocampus 6: $579-590$.

Lipton, P.A., White, J.A., and Eichenbaum, H. 2007. Disambiguation of overlapping experiences by neurons in the medial entorhinal cortex. I. Neurosci. 27: 5787-5795.

Louie, K. and Wilson, M.A. 2001. Temporally structured replay of awake hippocampal ensemble activity during rapid eye movement sleep. Neuron 29: 145-156.

Manns, J.R. and Eichenbaum, H. 2005. Time and treason to the trisynaptic teachings: Theoretical comment on Kesner et al. (2005). Behav. Neurosci. 119: 1140-1143.

Marr, D. 1971. Simple memory: A theory for archicortex. Philos. Trans. $R$ Soc. Lond. B Biol. Sci. 262: 23-81.

Maurer, A.P., Vanrhoads, S.R., Sutherland, G.R., Lipa, P., and McNaughton, B.L. 2005. Self-motion and the origin of differential spatial scaling along the septo-temporal axis of the hippocampus. Hippocampus 15: 841-852.

McNaughton, B.L. and Morris, R.G.M. 1987. Hippocampal synaptic enhancement and information storage within a distributed memory system. Trends Neurosci. 10: 408-415.

McNaughton, B.L. and Nadel, L. 1990. Hebb-Marr networks and the neurobiological representation of action in space. In Neuroscience and connectionist theory (eds. M.A. Gluck and D.E. Rumelhart), pp. 1-64. Lawrence Erlbaum Assoc., Hillsdale, NJ.

Mehta, M.R., Barnes, C.A., and McNaughton, B.L. 1997. Experience-dependent, asymmetric expansion of hippocampal place fields. Proc. Natl. Acad. Sci. 94: 8918-8921. 
Mehta, M.R., Lee, A.K., and Wilson, M.A. 2002. Role of experience and oscillations in transforming a rate code into a temporal code. Nature 417: 741-746.

Mitchell, S.J. and Ranck, J.B.J. 1980. Generation of theta rhythm in medial entorhinal cortex of freely moving rats. Brain Res. 178: $49-66$.

Mitchell, S.J., Rawlins, J.N., Steward, O., and Olton, D.S. 1982. Medial septal area lesions disrupt theta rhythm and cholinergic staining in medial entorhinal cortex and produce impaired radial arm maze behavior in rats. I. Neurosci. 2: 292-302.

O'Keefe, J. and Burgess, N. 2005. Dual phase and rate coding in hippocampal place cells: Theoretical significance and relationship to entorhinal grid cells. Hippocampus 15: 853-866.

O'Keefe, J. and Recce, M.L. 1993. Phase relationship between hippocampal place units and the EEG theta rhythm. Hippocampus 3: $317-330$.

O'Keefe, J., Burgess, N., Donnett, J.G., Jeffery, K.J., and Maguire, E.A. 1998. Place cells, navigational accuracy, and the human hippocampus. Philos. Trans. R. Soc. Lond. B Biol. Sci. 353: 1333-1340.

Olton, D.S., Becker, J.T., and Handelmann, G.E. 1979. Hippocampus, space and memory. Behav. Brain Sci. 2: 313-365.

Quirk, G.J., Muller, R.U., Kubie, J.L., and Ranck Jr., J.B. 1992. The positional firing properties of medial entorhinal neurons: Description and comparison with hippocampal place cells. J. Neurosci. 12: 1945-1963.

Rivas, J., Gaztelu, J.M., and Garcia-Austt, E. 1996. Changes in hippocampal cell discharge patterns and theta rhythm spectral properties as a function of walking velocity in the guinea pig. Exp. Brain Res. 108: 113-118.

Rogers, J.L., Hunsaker, M.R., and Kesner, R.P. 2006. Effects of ventral and dorsal CA1 subregional lesions on trace fear conditioning. Neurobiol. Learn. Mem. 86: 72-81.

Rolls, E.T. and Kesner, R.P. 2006. A computational theory of hippocampal function, and empirical tests of the theory. Prog. Neurobiol. 79: 1-48.

Rolls, E.T., Stringer, S.M., and Elliot, T. 2006. Entorhinal cortex grid cells can map to hippocampal place cells by competitive learning. Network 17: 447-465.

Sargolini, F., Fyhn, M., Hafting, T., McNaughton, B.L., Witter, M.P., Moser, M.B., and Moser, E.I. 2006. Conjunctive representation of position, direction, and velocity in entorhinal cortex. Science 312: 758-762.

Sato, N. and Yamaguchi, Y. 2003. Memory encoding by theta phase precession in the hippocampal network. Neural Comput. 15: 2379-2397.

Seager, M.A., Johnson, L.D., Chabot, E.S., Asaka, Y., and Berry, S.D. 2002. Oscillatory brain states and learning: Impact of hippocampal theta-contingent training. Proc. Natl. Acad. Sci. 99: 1616-1620.

Sharp, P.E. 1996. Multiple spatial/behavioral correlates for cells in the rat postsubiculum: Multiple regression analysis and comparison to other hippocampal areas. Cereb. Cortex 6: 238-259.

Sharp, P.E. and Turner-Williams, S. 2005. Movement-related correlates of single-cell activity in the medial mammillary nucleus of the rat during a pellet-chasing task. J. Neurophysiol. 94: 1920-1927.

Sharp, P.E., Turner-Williams, S., and Tuttle, S. 2006. Movement-related correlates of single cell activity in the interpeduncular nucleus and habenula of the rat during a pellet-chasing task. Behav. Brain Res. 166: $55-70$.

Slawinska, U. and Kasicki, S. 1998. The frequency of rat's hippocampal theta rhythm is related to the speed of locomotion. Brain Res. 796: $327-331$.
Solstad, T., Moser, E.I., and Einevoll, G.T. 2006. From grid cells to place cells: A mathematical model. Hippocampus 16: 1026-1031.

Stewart, M. and Fox, S.E. 1990. Do septal neurons pace the hippocampal theta rhythm? Trends Neurosci. 13: 163-168.

Stewart, M., Quirk, G.J., Barry, M., and Fox, S.E. 1992. Firing relations of medial entorhinal neurons to the hippocampal theta rhythm in urethane anesthetized and walking rats. Exp. Brain Res. 90: 21-28.

Tahvildari, B., Fransén, E., Alonso, A.A., and Hasselmo, M.E. 2007. Switching between "on" and "off" states of persistent activity in lateral entorhinal layer III neurons. Hippocampus 17: 257-263.

Taube, J.S., Muller, R.U., and Ranck Jr., J.B. 1990. Head-direction cells recorded from the postsubiculum in freely moving rats. I. Description and quantitative analysis. J. Neurosci. 10: 420-435.

Toth, K., Freund, T.F., and Miles, R. 1997. Disinhibition of rat hippocampal pyramidal cells by GABAergic afferent from the septum. J. Physiol. 500: 463-474.

Treves, A. and Rolls, E.T. 1994. Computational analysis of the role of the hippocampus in memory. Hippocampus 4: 374-391.

Tsodyks, M.V., Skaggs, W.E., Sejnowski, T.J., and McNaughton, B.L. 1996. Population dynamics and theta rhythm phase precession of hippocampal place cell firing: A spiking neuron model. Hippocampus 6: 271-280.

Vazdarjanova, A. and Guzowski, J.F. 2004. Differences in hippocampal neuronal population responses to modifications of an environmental context: Evidence for distinct, yet complementary, functions of CA3 and CA1 ensembles. J. Neurosci. 24: 6489-6496.

Vertes, R.P. and Kocsis, B. 1997.

Brainstem-diencephalo-septohippocampal systems controlling the theta rhythm of the hippocampus. Neuroscience 81: 893-926.

Wallenstein, G.V. and Hasselmo, M.E. 1997. GABAergic modulation of hippocampal population activity: Sequence learning, place field development, and the phase precession effect. J. Neurophysiol. 78: $393-408$.

Whishaw, I.Q. and Vanderwolf, C.H. 1973. Hippocampal EEG and behavior: Changes in amplitude and frequency of RSA (theta rhythm) associated with spontaneous and learned movement patterns in rats and cats. Behav. Biol. 8: 461-484.

White, J.A., Budde, T., and Kay, A.R. 1995. A bifurcation analysis of neuronal subthreshold oscillations. Biophys. J. 69: 1203-1217.

Winson, J. 1978. Loss of hippocampal theta rhythm results in spatial memory deficit in the rat. Science 201: 160-163.

Wood, E.R., Dudchenko, P.A., Robitsek, R.J., and Eichenbaum, H. 2000. Hippocampal neurons encode information about different types of memory episodes occurring in the same location. Neuron 27: 623-633.

Wyble, B.P., Hyman, J.M., Rossi, C.A., and Hasselmo, M. 2004. Analysis of theta power in hippocampal EEG during bar pressing and running behavior in rats during distinct behavioral contexts. Hippocampus 14: $368-384$.

Yamaguchi, Y. 2003. A theory of hippocampal memory based on theta phase precession. Biol. Cybern. 89: 1-9.

Yoon, T. and Otto, T. 2007. Differential contributions of dorsal vs. ventral hippocampus to auditory trace fear conditioning. Neurobiol. Learn. Mem. 87: 464-475.

Zilli, E.A. and Hasselmo, M.E. 2007. Modeling the role of working memory and episodic memory in behavioral tasks. Hippocampus (in press).

Received July 1, 2007; accepted in revised form September 24, 2007. 


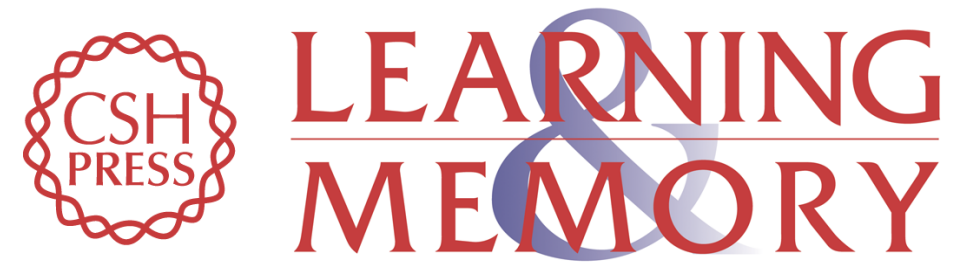

\section{Arc length coding by interference of theta frequency oscillations may underlie context-dependent hippocampal unit data and episodic memory function}

Michael E. Hasselmo

Learn. Mem. 2007, 14:

Access the most recent version at doi:10.1101//m.686607

References This article cites 104 articles, 22 of which can be accessed free at: http://learnmem.cshlp.org/content/14/11/782.full.html\#ref-list-1

License

Email Alerting

Receive free email alerts when new articles cite this article - sign up in the box at the Service top right corner of the article or click here. 